\title{
Transmission Property Analysis of Optically-Anisotropic Dielectric Multilayer for Thin Wide-Viewing-Angle Reflective Polarizer
}

\author{
Kunihiko AKAHANE ${ }^{\dagger}$, Takahiro ISHINABE ${ }^{\dagger a)}$, Members, Yosei SHIBATA ${ }^{\dagger}$, Nonmember, $^{\circ}$ \\ and Hideo FUJIKAKE ${ }^{\dagger}$, Fellow
}

SUMMARY We show that light leakage that occurs in reflective polarizers at large angles of incidence can be suppressed by using anisotropic dielectric multilayers with larger reflective indices in thickness direction and that the interference-included $2 \times 2$ Jones matrix method is useful for the investigation of the optical propagation properties of the dielectric multilayers. The thickness of the reflective polarizer can also be reduced by optimizing the distribution of the multilayers in the stack, whilst considering the visual sensitivity. These results indicate that it is possible to realize a high-quality liquid crystal display with wide viewing angles and high light utilization efficiency.

key words: reflective polarizer, anisotropic dielectric multilayers, wide viewing angle, film thickness distribution, thin thickness

\section{Introduction}

In recent years, noticeable progress in the optical performance of liquid crystal displays (LCDs) has been made due to the huge growth of smartphones and tablet terminals. The highest-resolution LCDs can be manufactured with over 500 pixels per inch (ppi) and have been demonstrated in practical use. However, high-definition LCDs have a low light utilization efficiency and aperture ratio because the thin film transistors (TFTs) and metal lines block light from the backlight, and have a fixed size regardless of the pixel size. Therefore, the improvement of the light utilization efficiency is the most important issue in the development of high-definition LCDs.

The reflective polarizer [1]-[8] has been used to improve the light utilization efficiency of LCDs. Figure 1 shows the operating principle of a reflective polarizer. The reflective polarizer is a dielectric multilayer film which is composed of thin polymer layers with alternating high and low refractive indices. The low-refractive-index polymer layer (L layer) is optically isotropic, whereas the high-refractive-index polymer layer (H layer) is uniaxially anisotropic. In this paper, the $\mathrm{x}$-axis is defined as the direction of the slow axis of the high-refractive-index layer (Fig. 1).

When the $\mathrm{L}$ layer and $\mathrm{H}$ layer both have the same refractive index in the y-axis ( $n_{\mathrm{Ly}}$ and $n_{\mathrm{Hy}}$, respectively), the reflective polarizer transmits the polarized light along the

Manuscript received March 1, 2017.

Manuscript revised May 11, 2017.

${ }^{\dagger}$ The authors are with Department of Electronic Engineering, Graduate School of Engineering, Tohoku University, Sendai-shi, 980-8579 Japan.

a)E-mail: ishinabe@ecei.tohoku.ac.jp

DOI: 10.1587/transele.E100.C.998

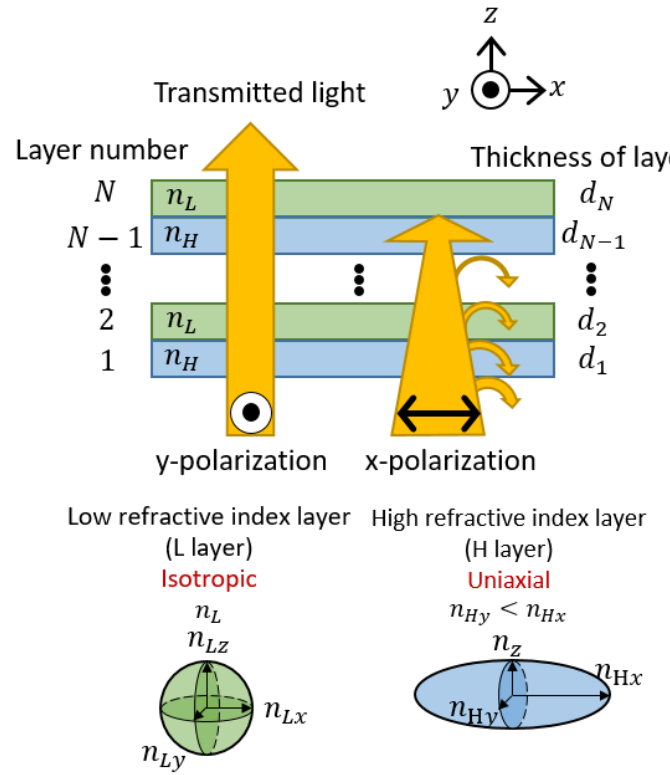

Fig. 1 Principle of reflective polarizer and refractive index ellipsoid of $\mathrm{L}$ and $\mathrm{H}$ layers.

$y$-axis (y-polarization). On the other hand, when the polarized light is aligned to the $\mathrm{x}$-axis (x-polarization), it is reflected due to the difference in refractive indices between the $\mathrm{L}$ and $\mathrm{H}$ layers ( $n_{\mathrm{Lx}}$ and $n_{\mathrm{Hx}}$, respectively). As a result, the transmitted light becomes linearly polarized along the $y-$ axis. The reflected $\mathrm{x}$-polarization light is reflected again and depolarized at the reflector that is located under the lightguide plate [9]. As a result, the reflected light can be reused as transmitted light and the light utilization efficiency is improved by 1.5 times compared with that of traditional LCDs.

The emitted light from backlight must pass through the reflective polarizer from a wide range of angles; therefore, the reflective polarizer must have no dependence on the incidence angle in order to achieve the highest utilization efficiency. However, in conventional reflective polarizers, the reflection wavelength is blue-shifted for incident light that is oblique in the $\mathrm{X}-\mathrm{z}$ plane. At large incidence angles, this means that longer-wavelength light will pass through, as shown in Fig. 2, which results in a color shift of the LCDs.

Figure 3 shows the measured chromaticity of the light transmitted through a reflective polarizer as a function of incidence angle. We measured the spectrum of the transmitted light by using Spectroradiometer (SR-UL1, Topcom 
Technohouse Corp.) and calculated the chromaticity in an xy-chromaticity diagram. We confirmed that the chromaticity of the transmitted light was red-shifted as the angle of incidence increased.

To solve this problem, increasing the layer thickness or the total number of layers has been proposed. By increasing the range of the reflection wavelength towards the longwavelength region, light leakage can be suppressed, even when the reflection wavelength band shifts towards shorter wavelengths. However, increasing the film thickness also increases the cost and complexity of the fabrication as the reflective polarizer is composed of several hundred polymer layers. To reduce the dependence of the incident angle in a reflective polarizer without increasing the thickness, it is necessary to clarify the optical propagation properties for the optically anisotropic dielectric multilayers with oblique light incidence. However, the optical properties for reflective polarizers with oblique incident light have not yet been fully elucidated.

In this paper, we examine the optical propagation properties of the dielectric multilayer with light at an oblique incidence and design a thin reflective polarizer with a wide viewing angle.

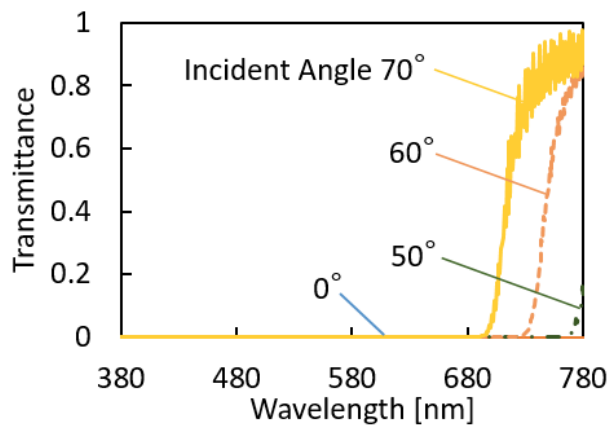

Fig. 2 Incident angle dependence of transmittance of reflective polarizer. (incident light: $\mathrm{x}$-polarization, incident plane: $\mathrm{x}-\mathrm{z}$ plane)

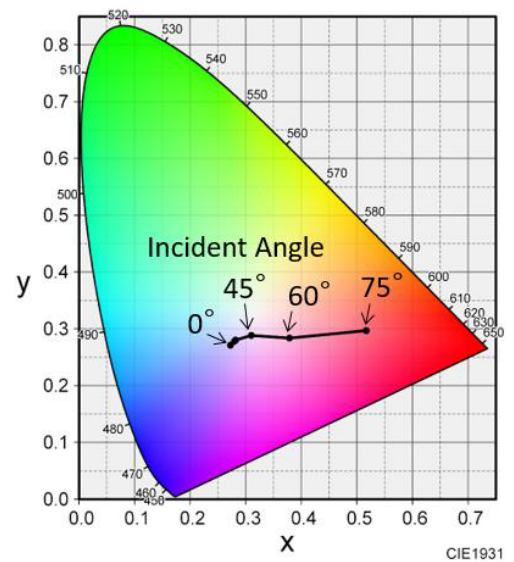

Fig. 3 Shift of chromaticity of transmitted light of reflective polarizer as a function of incident angle.

\section{Interference-Included 2 × Jones Matrix Method}

In this section, we discuss the method used to analyze the optical propagation properties of the optically anisotropic dielectric multilayers for oblique light incidence. In general, Berreman's $4 \times 4$ matrix method [10], [11] has been widely used to calculate optical propagation in dielectric multilayers. However, an analysis of the transmission properties for each layer is difficult because each matrix component includes the effects of light transmission, reflection, phase change, and interference, which cannot be treated separately.

The Interference-Included Jones Matrix Method (IIM method) [12], which is based on the extended $2 \times 2$ Jones matrix method [13]-[15], can also be used to calculate the transmittance of each layer by considering multiple reflections and multiple interference paths. In the IIM method, the transmission vector $\left(T_{\mathrm{x}}, T_{\mathrm{y}}\right)$ is calculated by multiplying the Jones matrix, $\mathbf{M}_{\mathrm{N}}$, of the medium by the incidence vector $\left(A_{\mathrm{x}}, A_{\mathrm{y}}\right)$, as shown in the equation:

$$
\left(\begin{array}{l}
T_{x} \\
T_{y}
\end{array}\right)=\mathbf{M}_{\mathrm{N}}\left(\begin{array}{l}
A_{x} \\
A_{y}
\end{array}\right) \quad(N \text { : number of layer })
$$

The Jones Matrix $\mathbf{M}_{\mathrm{N}}$ is a $2 \times 2$ matrix, which shows the optical characteristics of a multilayer film consisting of $N$ layers and is expressed as:

$$
\begin{aligned}
& \boldsymbol{M}_{N} \\
& =\boldsymbol{D}_{N} \Sigma \boldsymbol{G}_{N} \boldsymbol{P}_{N}^{+} \boldsymbol{D}_{N-1} \Sigma \boldsymbol{G}_{N-1} \boldsymbol{P}_{N-1}^{+} \boldsymbol{D}_{N-2} \cdots \Sigma \boldsymbol{G}_{1} \boldsymbol{P}_{1}^{+} \boldsymbol{D}_{0}
\end{aligned}
$$

where $\mathbf{D}_{\mathrm{N}}$ is a transmission matrix between layers $N$ and $N+1, \mathbf{P}_{\mathrm{N}}^{ \pm}$is the propagation matrix of the layer $N$ in the forward and backward directions, and $\mathbf{G}_{\mathrm{N}}$ is the interference matrix of the layer $N$ (see Fig. 4).

In the IIM Method, each optical phenomenon is represented by a different matrix and, hence, the IIM method is easier to analyze the optical characteristics of multilayer

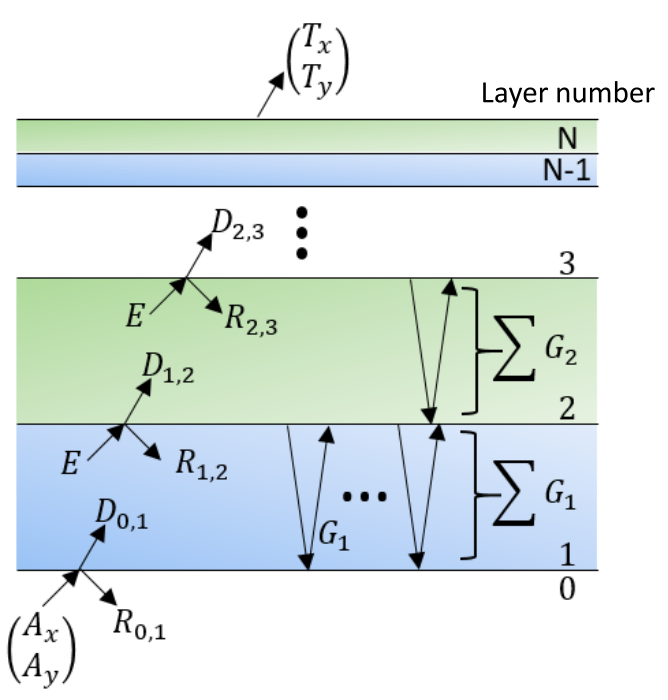

Fig. 4 Principle of IIM Method 
films compared to the $4 \times 4$ matrix method.

In the case of the reflective polarizer, incidence angle dependence arises with light polarized in the $\mathrm{x}$-axis; therefore, Eq. (1) could be simplified as follows:

$$
\left(\begin{array}{c}
T_{x} \\
0
\end{array}\right)=\boldsymbol{M}_{N}\left(\begin{array}{c}
A_{x} \\
0
\end{array}\right)=\left[\begin{array}{cc}
m_{[1,1]} & 0 \\
0 & m_{[2,2]}
\end{array}\right]\left(\begin{array}{c}
A_{x} \\
0
\end{array}\right)
$$

In Eq. (3), the [1,2] and [2,1] components of the Jones matrix $\mathbf{M}_{\mathrm{N}}$ become zero because the azimuth of the incident light is parallel to the slow axis of the $\mathrm{H}$ layer. It was found that the component $[1,1]$ was the only valid incidence vector component of $A_{\mathrm{x}}$ and should be solely focused on for the analysis and design of the reflective polarizer.

\section{Incidence Angle Dependence Analysis for the Reflec- tive Polarizer}

We analyzed the optical propagation properties of the reflective polarizer by using the $2 \times 2$ IIM method for reducing the incidence angle dependence. The incidence angle dependence of the transmitted light in a reflective polarizer arises only due to long-wavelength light. Therefore, we considered that the matrix component $[1,1]$ in Eq. (3) also changes with the angle of incidence. The matrix component $[1,1]$ in Eq. (3) is a multiplication of the matrix component $[1,1]$ of transmission, propagation and interference matrixes and is represented by:

$$
m_{[1,1]}=\prod\left(\sum G_{k[1,1]}\right) \prod P_{k[1,1]}^{+} \prod D_{k[1,1]}
$$

We considered that any matrix which increased in value for large incidence angles in the long-wavelength region could be the cause of incidence angle dependence of the matrix component $[1,1]$. For this reason, we calculated incidence angle dependence of each matrix component for $N$ multilayers. The calculation results are shown in Fig. 5.

As shown in Fig. 5 (a), the transmission matrix of the component $[1,1]$ decreases with increasing incidence angle in the long-wavelength range. The propagation matrix of the component $[1,1]$ does not change with the incidence angle (Fig. 5 (b)). These results show that the transmission and propagation matrix are not the cause of the angle dependence in the reflective polarizer. On the other hand, we found that the interference matrix of component $[1,1]$ increases for large incidence angles (Fig. 5 (c)).

As a result, we clarified that the cause of the incidence angle dependence of transmitted light in the reflective polarizer was the interference matrix. Based on this result, we further analyzed the optical characteristics of the interference matrix so that the incidence angle dependence could be improved. The interference matrix of component $[1,1]$ was expressed as follows:

$$
\mathbf{G}_{k[1,1]}=\boldsymbol{P}_{k[1,1]}^{+} \boldsymbol{R}_{\mathrm{k}[1,1]} \mathbf{P}_{\mathrm{k}[1,1]}^{-} \boldsymbol{R}_{\mathrm{k}[1,1]}
$$

where $\mathbf{R}$ is a reflection matrix.

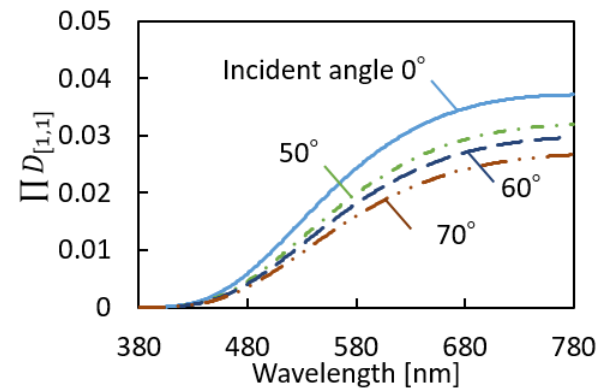

(a)

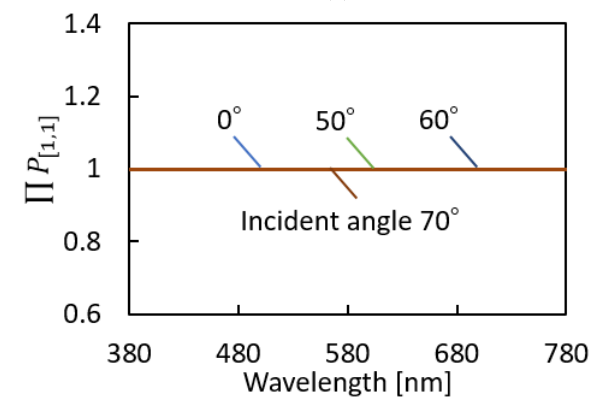

(b)

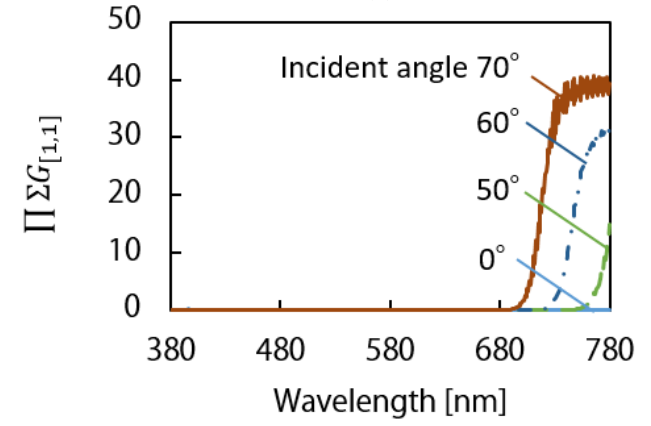

(c)

Fig. 5 The change of the wavelength property of component of (a) transmission (b) propagation, and (c) interference matrix as a function of incident angle.

In general, the phase change of each layer of the reflective polarizer is designed to be $\pi / 2$ for normally incident light in order to maximize the attenuation rate of light by the interference. Therefore, we considered that the incidence angle dependence of the interference matrix was due to a change of the value of phase change in each layer. The value of the phase change for each layer is included in the propagation matrix $\mathbf{P}$ in the IIM method. Therefore, we analyzed the propagation matrix to improve the incidence angle dependence of the transmitted light.

Equation (6) represents the component of propagation matrix in layer $k$.

$$
\mathrm{P}_{\mathrm{k}[1,1]}=e^{\left[\left(-k_{x z k} * d_{k}\right) i\right]}
$$

$k_{\mathrm{xz}}$ denotes the wavenumber of the x-polarization in the $\mathrm{z}$ direction, and $d$ represents the thickness of the layer. $k_{\mathrm{xz}}$ was calculated by:

$$
k_{x z} \cdot d=\frac{2 \pi}{\lambda} \sqrt{n_{x}^{2}-\frac{n_{x}^{2}}{n_{z}^{2}} \sin ^{2} \theta} \cdot d
$$


As shown in Eq. (7), it is evident that the incidence angle dependence of the propagation matrix depends on $n_{\mathrm{x}} / n_{z}$.

We clarified that the incidence angle dependence can be suppressed by increasing the refractive index along the $\mathrm{z}$ direction $\left(n_{\mathrm{z}}\right)$ to reduce the value of $\left(n_{\mathrm{x}} / n_{\mathrm{z}}\right)$. As a result, we proposed a new structure for a reflective polarizer that is composed of polymer layers with high refractive index along the $\mathrm{z}$ direction to reduce the incidence angle dependence. Figure 6 shows the incidence angle dependence of $k_{\mathrm{xz}} \cdot d$ for the conventional and proposed $\mathrm{H}$ layers at a wavelength of $700 \mathrm{~nm}$. Table 1 shows the refractive indices used for the calculation at $700 \mathrm{~nm}$.

As shown in Fig. 6, the change of $k_{x z} \cdot d$ for increasing angles of incidence in the proposed $\mathrm{H}$ layer was smaller than that of the conventional one. Figure 7 shows the transmission spectra of the proposed reflective polarizer as a function of incidence angle and the refractive index ellipsoids of the

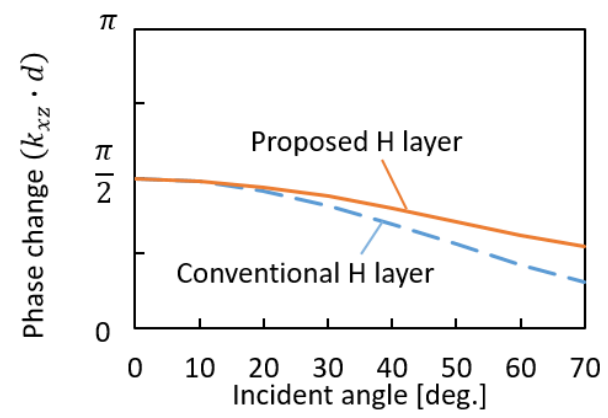

Fig. 6 Incident angle dependence of phase change $k_{\mathrm{xz}} \cdot d$. (Wavelength: $700 \mathrm{~nm})$

Table 1 Refractive indices of multilayers. (Wavelength: 700nm)

\begin{tabular}{|c|c|c|}
\hline & Conventional & Proposed \\
\hline & $n_{\mathrm{Hx}}=1.9$ & $n_{\mathrm{Hx}}=1.9$ \\
& $n_{\mathrm{Hy}}=1.6$ & $n_{\mathrm{Hy}}=1.6$ \\
& $n_{\mathrm{Hz}}=1.6$ & $n_{\mathrm{Hz}}=1.9$ \\
\hline \multirow{3}{*}{ L layer } & $n_{\mathrm{Lx}}=1.6$ & $n_{\mathrm{Lx}}=1.6$ \\
& $n_{\mathrm{Ly}}=1.6$ & $n_{\mathrm{Ly}}=1.6$ \\
& $n_{\mathrm{Lz}}=1.6$ & $n_{\mathrm{Lz}}=1.9$ \\
\hline
\end{tabular}

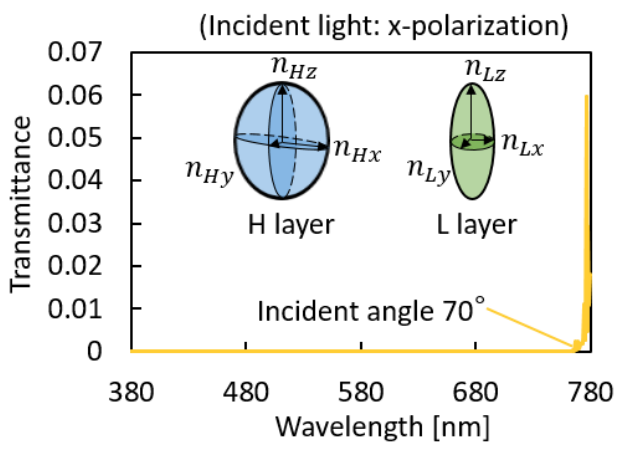

Fig. 7 Transmittance of the proposed reflective polarizer and the refractive index ellipsoids of the $\mathrm{H}$ and $\mathrm{L}$ layers.
$\mathrm{H}$ and $\mathrm{L}$ layers. The incidence light was x-polarized.

As shown in Fig. 2, the conventional reflective polarizer exhibits light leakage with an incidence angle of over $50^{\circ}$ in the long-wavelength range. In contrast, the proposed reflective polarizer shows no light leakage even for light with an angle of incidence of over $70^{\circ}$ (Fig. 7). As a result, we confirmed that we successfully suppressed the incidence angle dependence of transmitted light for reflective polarizers by increasing the refractive index along the $\mathrm{z}$ direction.

\section{Optimization of Film Thickness Distribution for Thin Reflective Polarizer}

Next, we investigated the optimum distribution of the polymer layer thicknesses to achieve the thin reflective polarizer. In general, the thickness distribution of a conventional reflective polarizer is a linear distribution [16]. In the linear thickness distribution, the film thickness of each layer gradually increases from the first layer to the last layer. Figure 8 shows the transmission spectra for a reflective polarizer with a linear thickness distribution and using $\mathrm{x}$-polarized incident light.

As shown in Fig. 8, the transmittance has wavelength dependence with a peak in transmittance at around $580 \mathrm{~nm}$, very close to the maximum sensitivity wavelength of the human eye $(\sim 550 \mathrm{~nm})$. Therefore, we considered that the number of layers could be reduced by removing the layers that correspond to the short- and long-wavelength ranges. However, when using a linear thickness distribution, reducing the total number of layers increases the transmittance throughout the entire visible range.

Therefore, we developed a polymer layer distribution model that would allow precise control of the intensity of the transmitted light in a narrow wavelength range. In general, the wavelength of the reflected light depends on the thickness and refractive index of the polymer layer. Equation (8) represents the relationship between the layer thickness and the principal wavelength, $\lambda$, for the reflected light.

$$
d=\frac{\lambda}{2\left(n_{L x \lambda}+n_{H x \lambda}\right)}
$$

$n_{\mathrm{Hx} \lambda}$ and $n_{\mathrm{Lx} \lambda}$ denote the refractive indices for the $\mathrm{H}$ and

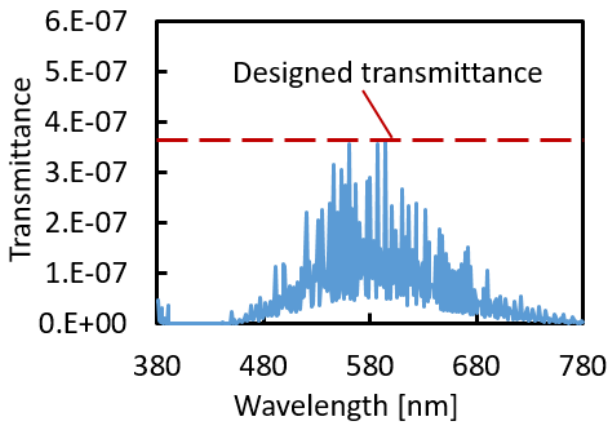

Fig. 8 Calculated transmission spectra of the polarizer using the linear thickness distribution. 


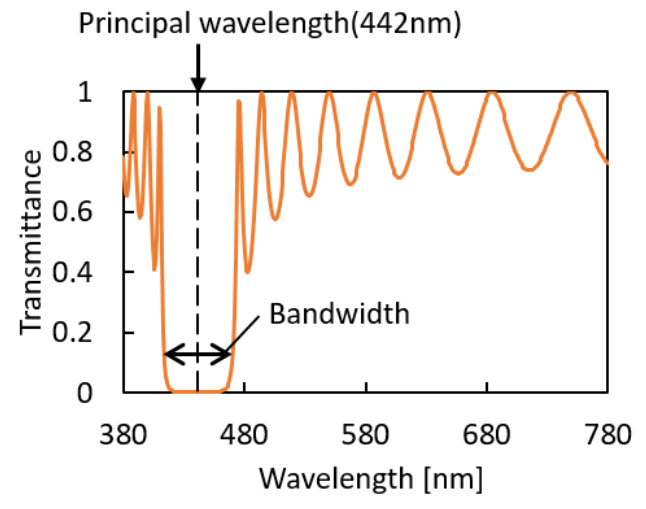

Fig.9 Transmission spectra of stacked layers (number of layers: 36, thickness of layer: $60.27 \mathrm{~nm}$ ).

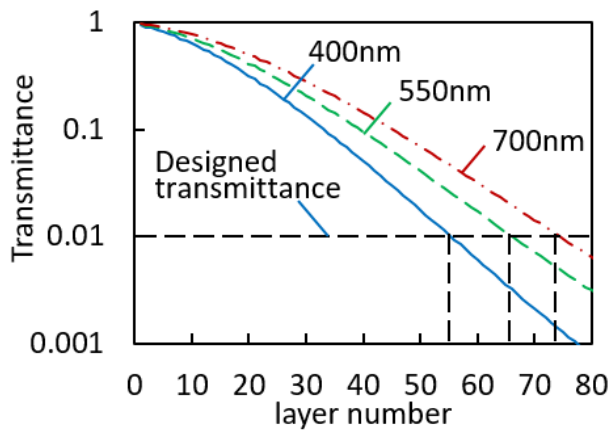

Fig. 10 Change of the transmittance of reflective polarizer as a function of layer number for different wavelengths.

L layers at the principal wavelength using light in the $\mathrm{x}$ polarization. According to Eq. (8), the transmittance of the principal wavelength decreases when polymer layers of the same thickness are stacked. Figure 9 shows the calculated result of the transmission spectra for one set of multilayers. The principal wavelength is $442 \mathrm{~nm}$.

As shown in Fig. 9, the transmittance becomes low for the principal wavelength and several tens of wavelengths either side of it. The bandwidth of the reflected light was $39 \mathrm{~nm}$. As a result, we confirmed that a set of multilayers having the same thickness could decrease transmittance in a narrow wavelength range. Additionally, we found that the transmittance can decrease multiple wavelengths at a time by using several sets of multilayers, each with a different principal wavelength. Therefore, we controlled the transmission spectra of a reflective polarizer by optimizing layer numbers for each set of multilayers. We derived the minimum number of layers necessary to decrease the transmittance to a value of 0.01 for three wavelengths $(400 \mathrm{~nm}$, $550 \mathrm{~nm}$, and $700 \mathrm{~nm}$ ) (Fig. 10).

As shown in Fig. 10, the number of layers required was different for each of the three wavelengths, where the numbers of layers were 56, 67, and 75 layers for $400 \mathrm{~nm}$, $550 \mathrm{~nm}$, and $700 \mathrm{~nm}$, respectively. This is because the difference in the refractive indices between the L layer and the $\mathrm{H}$ layer $\left(n_{\mathrm{Lx}}\right.$ and $n_{\mathrm{Hx}}$ ) becomes small at longer wavelengths

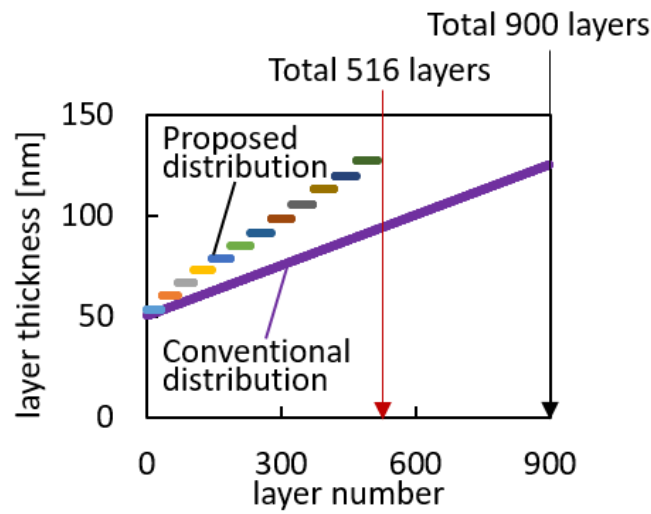

Fig. 11 Comparison between the conventional and proposed thickness distribution.

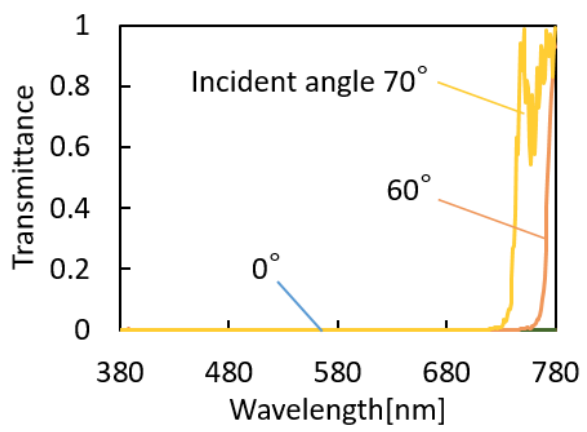

Fig. 12 Transmission spectra of thin reflective polarizer.

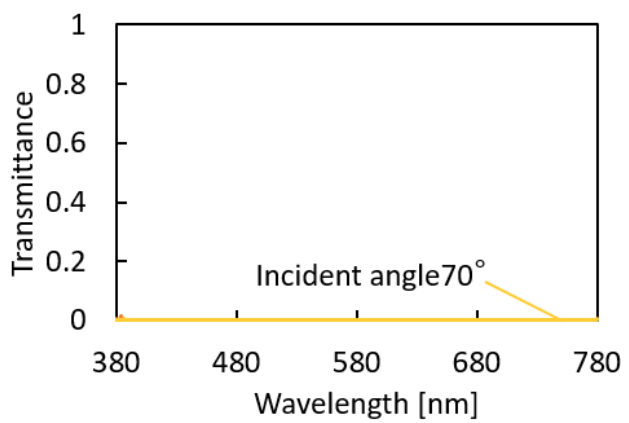

Fig. 13 Transmission spectra of Thin Wide-Viewing-Angle reflective polarizer.

due to the dispersion of refractive indices. Therefore, the thin reflective polarizer could be realized by using a unique thickness distribution for each specific wavelength, reducing the total number of layers in the structure. Figure $11 \mathrm{com}-$ pares the minimum number of multilayers required for each method, where the number of layers required in the conventional thickness distribution was 900 but in the proposed thickness distribution only 516 were needed. Consequently, the proposed thickness distribution can reduce the number of layers that are required in the reflective polarizer.

Furthermore, Fig. 12 shows the transmission spectra of the reflective polarizer using the proposed thickness distribution. We confirmed that the transmitted spectra using the 
proposed thickness distribution has the same transmission spectra as the conventional one. Finally, we calculated the transmission spectra of the reflective polarizer using the proposed thickness distribution and increased refractive index along the $\mathrm{z}$ direction of the $\mathrm{L}$ and $\mathrm{H}$ layers (Fig. 13). We confirmed that this novel reflective polarizer exhibited no light leakage, even at incidence angles in excess of $70^{\circ}$.

\section{Conclusion}

Using the IIM method, we analyzed the optical propagation properties of an optically anisotropic dielectric multilayer to suppress the incidence angle dependence of transmitted light. We confirmed that the matrix component $[1,1]$ was critical for the analysis and design of a reflective polarizer. We showed that, in a reflective polarizer, the incidence angle dependence of the transmitted light could be suppressed by increasing the refractive index of the $\mathrm{L}$ and $\mathrm{H}$ layers along the $\mathrm{z}$ direction. In addition, we confirmed that the thickness of the reflective polarizer could be reduced by optimizing the distribution of film thickness to control the transmittance in the narrow-wavelength range.

We successfully designed a thin reflective polarizer with a wide viewing angle. These results indicate that it is possible to realize high-quality liquid crystal displays with wide viewing angles and high light utilization efficiency.

\section{References}

[1] R. Zhu, G. Tan, J. Yuan, and S. Wu, "Functional reflective polarizer for augmented reality and color vision deficiency," vol.24, no.5, pp.2490-2495, 2016.

[2] M.F. Weber, C.A. Stover, L.R. Gilbert, T.J. Nevitt, and A.J. Ouderkirk, "Giant Birefringent Optics in Multilayer Polymer Mirrors," vol.287, no.5462, pp.2451-2456, 2000.

[3] A. History, "The Wire Grid as a Near-Infrared Polarizer," vol.50, no.1954, pp.886-891, 1956.

[4] M.Y. Yu, B.W. Lee, J.H. Lee, and J.H. Ko, "Correlation between the optical performance of the reflective polarizer and the structure of LCD backligeht," J. Opt. Soc. Korea, vol.13, no.2, pp.256-260, 2009.

[5] Z.N. Yu, P. Deshpande, W. Wu, J. Wang, and S.Y. Chou, "Reflective polarizer based on a stacked double-layer subwavelength metal grating structure fabricated using nanoimprint lithography," Appl. Phys. Lett., vol.77, no.7, pp.927-929, 2000.

[6] T. Kim, K. Kim, E.S. Lee, W.Y. Jeong, D.Y. Lim, and S.-W. Choi, "Reflective composite sheet design for LCD backlight recycling," Opt. Express, vol.22, no.9, p.11175, 2014.

[7] S.H. Kim, J.-D. Park, and K.-D. Lee, "Fabrication of a nano-wire grid polarizer for brightness enhancement in liquid crystal display," Nanotechnology, vol.17, no.17, pp.4436-4438, 2006.

[8] H.A.C. Hen and R.U.Z. Hu, "Enlarging the color gamut of liquid crystal displays with a functional reflective polarizer," vol.47, no.2014, pp.425-427, 2017.

[9] Z. Ge and S.T. Wu, "Nanowire grid polarizer for energy efficient and wide-view liquid crystal displays," Appl. Phys. Lett., vol.93, no.12, pp.3-5, 2008.

[10] D.W. Berreman, "Optics in Stratified and Anisotropic Media: 4x4-Matrix Formulation," J. Opt. Soc. Am., vol.62, no.4, pp.502-510, 1972 .

[11] H. Wöhler, G. Haas, M. Fritsch, and D.A. Mlynski, "Faster $4 \times 4$ matrix method for uniaxial inhomogeneous media," J. Opt. Soc. Am.
A, vol.5, no.9, p.1554, 1988.

[12] Y. Ohno, T. Ishinabe, T. Miyashita, and T. Uchida, "Highly Accurate Method for Measuring Ordinary and Extraordinary Refractive Indices of Liquid Crystal Materials, Cell Thickness, and Pretilt Angle of Liquid Crystal Cells Using Ellipsometry,' Jpn. J. Appl. Phys., vol.48, no.5, p.51502, 2009.

[13] R.C. Jones, "A New Calculus for the Treatment of Optical Systems," J.O.S.A., vol.31, no.7, pp.488-493, 1941.

[14] P. Yeh, "Extended Jones matrix method," vol.72, no.4, pp.507-513, 1982.

[15] C. Gu, "Extended Jones matrix method. II," vol.10, no.5, pp.966973, 1993.

[16] Y. Li, T.X. Wu, S. Member, and S. Wu, "Design Optimization of Reflective Polarizers for LCD Backlight Recycling," vol.5, no.8, pp.335-340, 2009.

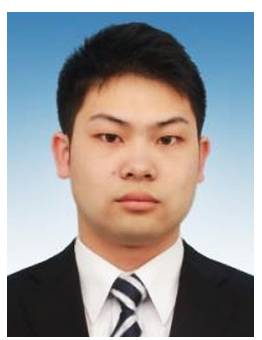

Kunihiko Akahane received the B.E. degree from Sendai National College of Technology Advanced Course, Sendai, Japan, in 2015. Now, he is a graduate student in the Department of Electronic Engineering, Graduate School of Engineering, Tohoku University. He is engaged in the flexible LCDs. He received the Outstanding Poster Paper Award from IDW in 2016.

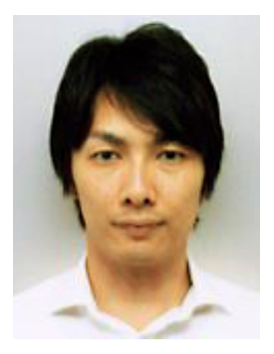

Takahiro Ishinabe received his B.S., M.S., and $\mathrm{Ph}$. D. degrees in Electronic Engineering from Tohoku University, Sendai, Japan, in 1995, 1997 and 2000, respectively. From 2000 to 2002, he was a Research Fellow of the Japan Society for the Promotion of Science and from 2003 to 2012, he was an Assistant Professor, and since 2013, he has been an Associate Professor in the Department of Electronics, Graduate school of Engineering, Tohoku University. He has also been a Visiting Professor in the CREOL, The College of Optics and Photonics, University of Central Florida from 2010 to 2011. He has been performing a research on advanced liquid crystal displays such as wide viewing angle $L C D$, reflective full-color LCD, field sequential color LCD and flexible LCD.

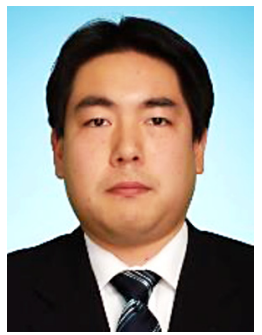

Yosei Shibata received his $\mathrm{PhD}$ degree in engineering from Tokyo Institute of Technology (Japan) in March 2013. He has research interests on organic semiconductor devices such as organic transistors and organic photovoltaics. Then he joined the National Institute of Advanced Industrial Science and Technologies (AIST, Japan) as a postdoctoral position. In October 2015, he joined the Department of Electronics of Tohoku University as an Assistant Professor. 


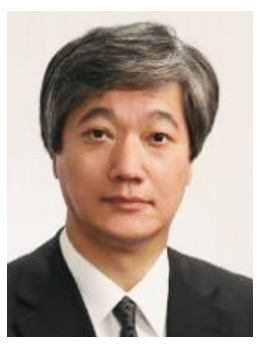

Hideo Fujikake received M.E and Ph.D. degrees from Tohoku University, Japan, in 1985 and 2003, respectively. In 1985, he joined Japan Broadcasting Corporation (NHK). In 19882012, he was with NHK Science and Technology Research Laboratories. Since 2012, he has been a professor at Department of Electronic Engineering, Tohoku University. He received Best Paper Award from Institute of Electronics, Information and Communication Engineers (IEICE) in 2001, Best Paper Award from Japanese Liquid Crystal Society (JLCS) in 2001 and 2015, NiwaTakayanagi Best Paper Awards from Institute of Image Information and Television Engineers of Japan (ITE) in 2003 and 2009, and Electronics Society Award from IEICE in 2013. His current interest is concerned with flexible liquid crystal displays. He also served as a General Vice Chair in International Display Workshops in 2015-2016, a Japan Chapter Chair in IEEE Consumer Electronics Society in 2012-2014, and a Vice President of Japanese Liquid Crystal Society in 2015-2016. He is an IEICE fellow since 2015, and ITE fellow since 2016. 